\title{
Treuhand transdisziplinär: Deformation, Disruption, Dynamik, Devaluation
}

\begin{abstract}
Die Geschichte des wirtschaftlichen Wiederaufbaus im post-sozialistischen Ostdeutschland nach 1990 ist vor allem mit der viel diskutierten Treuhandanstalt verbunden. Verschiedene Narrative beleuchten langfristige strukturelle Veränderungen, kurzfristige Brüche, mittelfristige Dynamiken sowie die Rahmung kultureller Reflexionen auf individueller oder kollektiver Ebene. Diese Muster könnten nicht nur helfen, heuristische Brücken zwischen verschiedenen Disziplinen wie Sozialwissenschaften, Ökonomie oder Geschichte in diesem neu aufkommenden Wissenschaftsbereich zu bauen, sondern auch dazu beitragen, diese Forschung in breitere Themen auf europäischer oder sogar globaler Ebene einzubetten.
\end{abstract}

Die Treuhand ist wieder da. War diese in den früheren 1990er-Jahren hochumstrittene Organisation nach der Jahrtausendwende weitgehend aus dem Fokus der nationalen politischen, medialen und auch wissenschaftlichen Debatten verschwunden, kehrte die Treuhandanstalt ab 2015 und vor allem auch seit 2019 wieder massiv auf die diversen Agenden und in verschiedenen Arenen zurück: In den Landtagswahlkämpfen des Jahres 2019 forderten etwa die Linke sowie später auch die AfD vergeblich die Einsetzung neuer parlamentarischer Untersuchungsausschüsse zur Treuhand. Daneben war der Themenkreis Treuhand, Wirtschaftsumbau sowie deren sozioökonomische und soziokulturelle Folgen Gegenstand etlicher Konferenzen, Diskussionsrunden und Podiumsveranstaltungen - mithin also umfassender zivilgesellschaftlicher Debatten, allerdings vorwiegend in Ostdeutschland selbst. Auch diverse Kunstprojekte vor allem jüngerer Künstler:innen, etwa Theaterstücke, Videoinstallationen oder andere Projektaktionen, haben dieses lange Zeit wenig beachtete Thema neuerlich umfassend aufgegriffen. Schließlich waren es auch über ein halbes Dutzend größerer Medienproduktionen, die ab 2018 die Treuhand zum Thema hatten - bis hin zur jüngsten, kontrovers aufgenommenen

(C) Der/die Autor:in(nen) 2021. Open Access: Dieser Artikel wird unter der Creative Commons Namensnennung 4.0 International Lizenz veröffentlicht (creativecommons.org/licenses/by/4.0/deed.de)

Open Access wird durch die ZBW - Leibniz-Informationszentrum Wirtschaft gefördert.

Dr. Marcus Böick, ist Akademischer Rat an der Fakultät für Geschichtswissenschaft der RuhrUniversität Bochum.
Netflix-Serie „Rohwedder. Einigkeit und Mord und Freiheit“ (Böick und Goschler, 2017; Köpping, 2018; Pötzl, 2019).

In der Sphäre der verschiedenen wissenschaftlichen Disziplinen lässt sich auch eine Rückkehr der Treuhand als Forschungsthema beobachten. Insbesondere die Sozial-, Wirtschafts- und Geschichtswissenschaften haben sich, gestützt unter anderem durch erhebliche, auch gezielt mobilisierte Fördermittel, des Themas erneut angenommen. Die sukzessive Erschließung des umfassenden, mehr als 35 Regalkilometer Akten umfassenden Treuhand-Bestands durch das Bundesarchiv in Berlin hat diese neuerliche Konjunktur in diversen Disziplinen erheblich befeuert. Dabei scheint die Aufgabenteilung geradezu paradigmatisch zu sein: Während sich die Sozial- und Kulturwissenschaften vor allem auf einer Mikroebene an den subjektiven Verarbeitungen und Reflexionen der Umbrüche (insbesondere durch betroffene Ostdeutsche aus diversen sozialen Gruppen und Alterskohorten) orientieren, widmet sich die akademische Ökonomie auf der Grundlage „harter" statistischer Daten (vor allem zu Privatisierungsprozessen in Unternehmen) einer bisweilen komplexen Modellbildung aus einer Makroperspektive. Demgegenüber finden zeithistorische Analysen ihren ebenfalls traditionellen Ansatzpunkt im institutionell produzierten und archivierten Quellenmaterial der Treuhand und anderer Verwaltungen, mit dessen Hilfe politische wie gesellschaftliche Kontexte sowie branchenbezogene Fall- sowie vergleichende Regionalstudien auf einer mittleren Ebene differenziert erarbeitet werden können (Hoffmann, 2020).

Die Treuhand und der von ihr wesentlich gelenkte und gestaltete Wirtschaftsumbau sind also tatsächlich auch in der Wissenschaft wieder aufgetaucht und werden entlang traditioneller Disziplingrenzen „aufgearbeitet“. Doch was folgt daraus für oft eingeforderte, aber meist nur selten wirklich realisierte inter- oder gar transdisziplinäre Perspektiv- 
setzungen? Alle sich gegenwärtig in dieses geschichtspolitisch wie erinnerungskulturell erheblich vorbelastete Feld vorwagenden Disziplinen müssen sich zunächst mit einer fast unüberschaubaren Gemengelage an zum Teil sehr polaren Deutungen und zugespitzten Interpretationen abarbeiten. Nicht nur die medial präsenten Skandale oder die politisch lange Zeit zwischen alternativlosen Heldenund neoliberalen Opfergeschichten schwankenden Narrative hallen dabei noch immer durch Diskussionshallen und Chaträume. Es sind insbesondere auch die umfassenden Diagnosen und Befunde der zeitgenössischen Transformations- und Treuhandforschung selbst, die es zugleich produktiv mitzudenken gilt - vor allem aus Ökonomie, Sozial- und Politikwissenschaft oder Rechtswissenschaft. Exemplarisch stehen hierfür die Arbeiten langjähriger Treuhand-Forschender wie dem Politikwissenschaftler Roland Czada (1993) oder dem Verwaltungswissenschaftler Wolfgang Seibel (2005) (Best und Holtmann, 2012).

Doch jenseits dieser geteilten wissenschaftlichen Vorarbeiten sowie der gesellschaftspolitischen Aufladungen des Themas scheinen die verschiedenen Disziplinen derzeit nur schwer in einen übergreifenden Dialog zu finden - zu unterschiedlich erscheinen die Prämissen, Methoden und Theorien sowie auch die Netzwerke und Fachkulturen. In diesem Essay möchte ich thesenartig vier wesentliche (und bewusst abstrakt gehaltene) Erzählperspektiven zur Diskussion stellen, die als übergreifende Ansatz- und Ausgangspunkte für gemeinsame Debatten zwischen den oft weit voneinander entfernten Disziplinen dienen könnten. Diese sind als Angebote zu verstehen, den oft sperrigfestgefahrenen Themenkomplex nicht nur interdisziplinär in den Blick zu nehmen, sondern diesen letztlich auch aus der meist einseitigen nationalstaatlichen Fokussierung, Fixierung und Fetischisierung herauslösen zu können.

\section{Perspektive I: Deformationen von Strukturen?}

Die bereits von den Zeitgenossen intensiv erörterte Frage, inwiefern die mentalen oder materiellen „Erblasten“ von DDR-Planwirtschaft und alltäglichem Realsozialismus maßgeblichen oder gar determinierenden Einfluss auf die Resultate des späteren Wirtschaftsumbaus hatten, steht bis in die Gegenwart im Fokus vielfältiger und meist sehr emotionaler Kontroversen: War in der DDR und ihrer zentral gesteuerten Industrielandschaft mit inren gigantischen Kombinaten alles „Schrott“? Waren ihre Bewohner:innen durch jahrzehntelange Sozialismuserfahrungen zudem auch mental „,deformiert“, wie Treuhand-Chef Detlev Rohwedder noch im Herbst 1990 so provokant wie zugespitzt formuliert hatte (Böick, 2018)? Oder hatte die DDR als vermeintlich führende "Industrienation" durchaus auch einige „Perlen“ sowie Fähigkeiten ihres „Humankapitals“ im Angebot, die sie in die gesamtdeutsche Ehe einzubringen vermochte? Etwas dezenter formuliert, geht es mithin in den teils sehr polemischen Diskussionen um die auch in hohem Maße symbolisch aufgeladene Frage, was die DDR, inre Wirtschaft, ihre Betriebe und letztlich insbesondere auch die in ihr lebenden Menschen eigentlich nach 1990 „wert“ waren (Steiner, 2007; Kemmler, 1994).

Dieses materiell-kulturelle Fragenbündel erweist sich, so meine These, als mit wissenschaftlichen Methoden schwerlich diskutier- oder entscheidbar. Dennoch schiene es ins Abstrakte gewendet lohnenswert, die möglichen bzw. unmöglichen Kontinuitäten langfristiger Strukturen und Mentalitäten nicht nur in Ost-, sondern auch in Westdeutschland über die vermeintlich scharf gezogene Zäsur von 1989/90 systematisch und in ihren jeweiligen Verschränkungen und Verwandlungen zu untersuchen. Eine derart langfristig angelegte Leitperspektive wurde bzw. wird in einigen, vornehmlich zeithistorischen Projekten bereits mit Erfolg erprobt (Brückweh et al., 2020). Dennoch läge an dieser Stelle noch erhebliches Forschungs- und Diskussionspotenzial für verschiedene Disziplinen. Der erste Narrativblock verortet also das nach 1989/90 stattgefundene Umbruchsgeschehen in sehr langen, historischen Linien, oder besser: materiellen Strukturen und kulturellen Mentalitäten.

\section{Perspektive II: Disruptionen durch Schocks?}

Allein für sich stehend wird ein derart langfristig-strukturfunktionaler Zugriff jedoch kaum befriedigen oder zu fachübergreifenden Diskussionen anregen können. Das trifft auch auf den verdichteten und überdeuteten Ereigniskomplex „1989/90“ zu, bei dem sich langfristige Konstellationen und kurzfristige Eruptionen in charakteristischer (wie durch die Zeitgenoss:innen in Ost und West weitgehend unvorhergesehener) Weise miteinander verbanden. Intensiv und kontrovers wird also das disruptive Element des Jahres 1989/90 im deutsch-deutschen Sonderfall diskutiert - insbesondere in Form der schnellen ökonomischen und politischen Vereinigung der beiden Staaten, Ökonomien und Gesellschaften in Form des „Beitritts“ bzw. der „Übertragung“ des westlichen Modells auf den Osten. Dies wird einerseits als besonders radikale Form einer "Schocktherapie" aus einer linken, kapitalismus- oder globalisierungskritischen Sichtweise attackiert. Andererseits verweisen sowohl konservative als auch liberale Interpret:innen (und damals aktive Zeitzeug:innen) verteidigend auf die vermeintliche „Alternativlosigkeit" der 1989/90 ohne größere Vorbereitungen, unter erheblichen Druck, unklaren äußeren Umständen sowie in großer Eile getroffenen Entscheidungen (Rödder, 2009).

Jenseits dieser kritischen wie affirmativen Bezugnahmen soll hier generell für einen übergreifenden Blick auf das Phänomen der Disruption geworben werden. Die freisetzende, entfesselnde Dynamik des im Herbst 1989/90 stimmen- 
und bildgewaltig einsetzenden Prozesses einer „Friedlichen Revolution" nicht nur in der DDR, sondern bereits zuvor in ganz Mittelosteuropa, wäre vielmehr als Initialzündung in einer Kette von mehr oder weniger tiefen Schocks, Einschnitten und Umbrüchen zu sehen, die auf die behäbigen Strukturen und Mentalitäten der insgesamt in Ost-WestKonfigurationen festgezurrten Staaten, Gesellschaften und Kulturen Europas einwirkten. Ein sehr klassisches, auf politische Aspekte verengtes Revolutionsverständnis verstellt den Blick auf die erheblichen disruptiven Dynamiken des Jahres 1989/90; auch breitere technologische wie globale Entwicklungstendenzen verstärkten den kurzfristig-disruptiven Effekt der dann doch wieder schärfer gefassten Zäsur nochmals. Im deutsch-deutschen Fall fand dieser disruptive Moment seinen deutlichsten Ausdruck in der über Nacht (und gegen das Votum vieler Ökonom:innen) vollzogenen Wirtschafts-, Währungs- und Sozialunion im Juli 1990.

\section{Perspektive III: Dynamisierung durch Beschleunigung?}

"Struktur" und „Schock“ können als zwei tragende narrative Elemente der bisherigen Debatten angesprochen werden. Doch nicht nur lang- und kurzfristige Faktoren wirkten zusammen. Auch hier könnte der bereits intensiv geführte Streit um die Geschwindigkeit der postsozialistischen Umgestaltungsprozesse ein Ausgangspunkt für weiterführende Überlegungen sein: Die Frage etwa, ob die im Osten oftmals massiv unter langfristigen Strukturdefiziten (der Planwirtschaft) und kurzfristigen Disruptionen (der Währungsunion) notleidenden Betriebe möglichst schnell entstaatlicht (also privatisiert oder stillgelegt) werden sollten - oder eben mehr Zeit für eine umfassende Sanierung, also eine Anpassung an die neuen Regeln und den intensiven Wettbewerb benötigen würden. Während die Bundesregierung und auch die Treuhand-Spitze den Weg massiv beschleunigter Privatisierungen („als wirksamster Form der Sanierung“, so Rohwedder) als beste Lösungsvariante für den Umbau der Ostwirtschaft ansahen, hielten kritische Stimmen aus den Reihen der linken Oppositionsparteien und der Gewerkschaften dagegen, dass man sowohl den Betrieben als auch den dort noch beschäftigten Menschen deutlich mehr Zeit zur unternehmerischen Sanierung und kulturellen Anpassung an die Marktwirtschaft einräumen müsse. Der erbitterte Grundsatzstreit „Privatisierung versus Sanierung“ wurde folglich zwischen den Polen „Ordnungspolitik“ und „Industriepolitik“ ausgefochten. Während der Privatisierungsprimat in den Jahren 1991/92 deutlich dominanter war, verdichtete sich nach 1993/94 ein auch bis in die CDU reichender Widerstand zur Vermeidung von „Deindustrialisierung“ und zum Erhalt „Industrieller Kerne“ (Böick und Lorke, 2019).

An diesem Punkt erweist sich der zeitgenössische Grundsatzstreit über die (zu hohe oder aber zu geringe) Geschwindigkeit der Transformationsprozesse ebenso als fruchtba- rer Ansatzpunkt weitergehender Reflexionen. Die sich bei allen Akteuren stark wandelnden Erfahrungsräume und Erwartungshorizonte oder simpler: das Gefühl, Teil eines „,historisch einmaligen" Vorgangs zu sein, bei dem sich binnen kürzester Zeit zuvor unvorstellbare Dinge in schneller Abfolge für immer veränderten, eröffnet eine weitere Blickachse. Dieser Blick ist stärker auf verschiedene Prozesse, Wandlungen und Zeitrhythmen ausgerichtet, innerhalb derer die Wechselbeziehungen von Vergangenheitsdeutungen, Gegenwartsanalysen und Zukunftsvorstellungen neu konfiguriert wurden. Sowohl materielle Prozesse als auch kulturelle Erfahrungen von mittelfristigen Be- und Entschleunigungen, also von gewandelten Zeitvorstellungen, erweisen sich insbesondere vor, während sowie nach 1990 als interessante analytische Perspektiven - und verknüpfen langfristige Strukturen mit kurzfristigen Disruptionen.

\section{Perspektive IV: Devaluationen durch Entwertungen?}

Eine vierte und letzte hier angerissene Facette schließt an eine ebenfalls bis in die Gegenwart hinein geführte Diskussion um Prozesse externer Um- und Entwertungen auf kollektiver wie auf individueller Ebene an. Hier ist es vor allem die dramatische weitgehende „Entwertung“ individueller Arbeits- und Lebensbiografien sowie kollektiver Arbeits- und Lebenswelten nach 1990, die vor allem von politischen, aber auch wissenschaftlichen Kritisierenden des „Einigungsprozesses“ wiederholt herausgestellt wurde. Die Ostdeutschen erschienen in diesem Deutungskontext als weitgehend passive Objekte und „Opfer" der umfassenden Umbrüche, während die Westdeutschen, vornehmlich als neue Führungskräfte bzw. „Transfereliten“ in die „neuen Bundesländer" kommend, als dominante sowie deutungs- und gestaltungsmächtige Akteure beschrieben werden. Dies verweist zugleich auch auf Diskussionen über eine mögliche „Kolonisierung“ des Ostens durch den Westen - in materieller wie kultureller Hinsicht. Dabei werden die fast vollständige Übertragung westlicher Regeln, die sukzessive Einsetzung westlicher Eliten sowie die weitgehende Übernahme des früheren Staatseigentums vorwiegend durch westliche Unternehmen überwiegend kritisch als integrale Bestandteile eines derartigen Szenarios einer „Übernahme“ gedeutet (Mau, 2019; Kowalczuk, 2019).

Auch hier bieten zeitgenössische Kontroversen Ansatzpunkte für analytische Perspektiven, die verschiedene Disziplinen zusammenführen können - versteht man das Setting eher als Prozess individueller und kollektiver Verund Bearbeitungen eines Umbruchs. Die Vermittlung und Verhandlung vergangener, gegenwärtiger wie zukünftiger Realitäten und Identitäten erwies sich dabei durchaus als grundlegender Katalog an Operationen einer intensiven Selbst- und Fremdreflexion von Akteuren im Umbruch über den Umbruch selbst. Diese übergreifenden Reorientie- 
rungsbemühungen fanden dabei keineswegs nur im Osten oder in der Wirtschaft statt, auch wenn sie hier besonders dramatisch sichtbar wurden. Sie strahlten letztlich - zumal nach dem Ende des Ost-West-Systemwettstreits - auf nahezu sämtliche gesellschaftliche Sphären aus. Diese intensiven Umdeutungen und Reflexionen umfassend in den Blick zu nehmen, würde schließlich auch weiterführende produktive Einblicke in die rahmenden Meta-Strukturen derartiger Transformationsprozesse ermöglichen.

\section{Fazit: neue Transformationsforschung als Gesellschaftswissenschaft}

Diese sehr experimentelle Skizze möchte Vorschläge für einen inter- oder gar transdisziplinären Dialog machen. Die in den letzten Jahren wieder massiv einsetzende ökonomische, sozialwissenschaftliche und zeithistorische Transformationsforschung sollte sich nicht in einem projektbezogenen aufmerksamkeitsgetriebenen Nebeneinander von vielen unverbundenen Einzelprojekten erschöpfen. Vier mögliche Ansatzpunkte für übergreifende Diskussionen wurden in diesem Rahmen zur Diskussion gestellt. Ein weiterführendes Nachdenken über langfristige Strukturen, kurzfristige Disruptionen, mittelfristige Dynamiken sowie metaperspektivische Reflexionen hat demonstriert, dass eingefahrene zeitgenössische Kontroversen durchaus auch in fruchtbar-produktive analytische Leitperspektiven transformiert werden können. Als heuristische Impulse können, so die Hoffnung, relativ abstrakte Begriffe und offene Konzepte bereitstellen, die eine gemeinsame Diskussion auch zwischen sehr unterschiedlichen Fächergruppen ermöglichen - im Sinne einer Transformationsforschung als übergreifender Gesellschaftswissenschaft (Böick et al., 2020).

Verstanden als möglichst abstrakt-offene Perspektiven bzw. „Slots“ können diese als mögliche Anschlusspunkte diskursive Räume zwischen den verschiedenen wissenschaftlichen Disziplinen für komplexe, differenzierte Analysen und Narrative aufspannen und eröffnen. Dies sollte auch als Aufforderung verstanden werden, den Blickwinkel unserer Analysen umfassend auszuweiten: Erstens etwa um auch die vielfältigen Akteure, Felder, Organisationen und Phänomene jenseits von Treuhand und Wirtschaftsumbau in den Blick zu nehmen. Zweitens um die Blickrichtung auch stärker als bislang systematisch vergleichend bzw. referenzierend auf die postsozialistischen Transformationsgesellschaften Mittelosteuropas zu richten. Drittens um, im Gegenschnitt, Wandlungsprozesse in der vermeintlich stabilen Welt des Westens anzusprechen, die Philipp Ther (2014) als „Ko-Transformationen“ bezeichnet hat. Viertens schließlich auch um Ansatzpunkte auf europäischer und letztlich globaler Ebene zu entwickeln, die sich mit der Genese und dem Zusammenwirken übergreifender Konstellationen (wie Digitalisierung und Globalisierung) beschäftigen. Kurzum: Die Treuhand ist also durchaus wieder da wir sollten aber als Wissenschaftler:innen nicht einfach bei ihr stehenbleiben (Krastev und Holmes, 2019).

\section{Literatur}

Best, H. und E. Holtmann (Hrsg.) (2012), Aufbruch der entsicherten Gesellschaft: Deutschland nach der Wiedervereinigung, Campus.

Brückweh, K., C. Villinger und K. Zöller (Hrsg.) (2020), Die lange Geschichte der „Wende“, Geschichtswissenschaft im Dialog, C. Links Verlag.

Böick, M. und C. Goschler (2017), Wahrnehmung und Bewertung der Arbeit der Treuhandanstalt, Bundesministerium für Wirtschaft und Energie.

Böick, M. (2018), Die Treuhand: Idee-Praxis, Wallstein Verlag.

Böick, M. und C. Lorke (2019), Aufschwung, Abbau, Anpassung? Eine kleine Geschichte des „Aufbau Ost“, in: Aus Politik und Zeitgeschichte, 46 (2019), 32-40

Böick, M., C. Goschler und R. Jessen (Hrsg.) (2020), Jahrbuch Deutsche Einheit, 1, C. Links.

Czada, R. (1993), Die Treuhandanstalt im Umfeld von Politik und Verbänden, in: W. Fischer, H. Hax und H.-K. Schneider (Hrsg.) (1993), Treuhandanstalt: Das Unmögliche wagen, Akademie Verlag, 148-173.

Hoffmann, D. (Hrsg.) (2020), Transformation einer Volkswirtschaft, Neue Forschungen zur Geschichte der Treuhandanstalt, Metropol.

Kemmler, M. (1994), Die Entstehung der Treuhandanstalt: Von der Wahrung zur Privatisierung des DDR-Volkseigentums, Campus.

Köpping, P. (2018), „Integriert doch erst mal uns!" Eine Streitschrift für den Osten, C. Links Verlag.

Kowalczuk, I.-S. (2019), Die Übernahme. Wie Ostdeutschland Teil der Bundesrepublik wurde, C.H. Beck.

Krastev, I. und S. Holmes (2019), The Light that Failed. A Reckoning, Allen Lane.

Mau, S. (2019), Lütten Klein. Leben in der ostdeutschen Transformationsgesellschaft, Suhrkamp.

Pötzl, N. F. (2019), Der Treuhand-Komplex. Legenden. Fakten. Emotionen, Kursbuch.

Rödder, A. (2009), Deutschland einig Vaterland: Die Geschichte der Wiedervereinigung, C.H. Beck.

Seibel, W. (2005), Verwaltete Illusionen: Die Privatisierung der DDR-Wirtschaft durch die Treuhandanstalt und ihre Nachfolger 1990-2000, Campus.

Steiner, A. (2007), Von Plan zu Plan: Eine Wirtschaftsgeschichte der DDR, Aufbau Taschenbuch Verlag.

Ther, P. (2014), Die neue Ordnung auf dem alten Kontinent: Eine Geschichte des neoliberalen Europa, Suhrkamp Verlag.

Title: Treuhand Transdisciplinary: Deformation, Disruption, Dynamics, Devaluation

Abstract: This paper examines new options regarding interdisciplinary perspectives on the history of economic reconstructions in post socialist Eastern Germany after 1990, mostly connected to the muchdebated Treuhandanstalt (Trusteeship Agency). By discussing different narratives, a brief, abstract framework is presented by highlighting longterm structural changes, shortterm disruptions, midterm dynamics as well as framing cultural reflections on an individual or collective level. These patterns could help to build heuristic bridges between different disciplines (like social sciences, economics, or history) in this re-emerging scientific area and to embed this research in broader themes on a European or even global scale.

JEL Classification: N94, O4, P2, R1 